\title{
BROKEN BRACELETS, MOLIEN SERIES, PARAFFIN WAX AND AN ELLIPTIC CURVE OF CONDUCTOR 48
}

\author{
TEWODROS AMDEBERHAN, MAHIR BILLEN CAN, AND VICTOR H. MOLL
}

\begin{abstract}
Aвstract. This paper introduces the concept of necklace binomial coefficients motivated by the enumeration of a special type of sequences. Several properties of these coefficients are described, including a connection between their roots and an elliptic curve. Further links are given to a physical model from quantum mechanical supersymmetry as well as properties of alkane molecules in chemistry.
\end{abstract}

\section{InTRODUCTION}

A jeweler is asked to design a necklace consisting of a chain with $n$ placements for $k$ pieces of diamond. The client ask for one group of $r$ diamonds to be placed next to each other and the remaining diamonds are to be isolated, that is, each one is mounted so that the two adjacent places are left empty. These special diamonds are called the medallion of the necklace. Figure 1 shows a necklace of length 20 , with a medallion of length 5 and four extra diamonds.

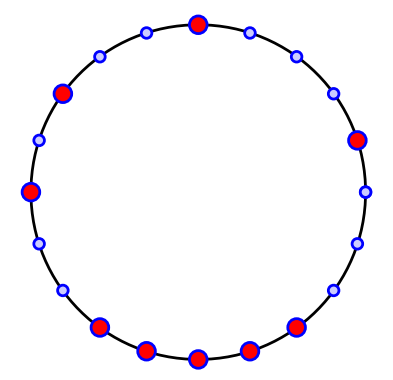

Figure 1. A necklace with a medallion.

Throughout this paper, a necklace is understood to be cyclically symmetric (unlabelled) formed by diamonds of two colors (i.e., binary).

Date: October 13, 2018.

2000 Mathematics Subject Classification. Primary 05A15, 05E10, Secondary 06A07, 06F05, 20M32.

Key words and phrases. necklaces, elliptic curves, Molien series, zeros of polynomials. 


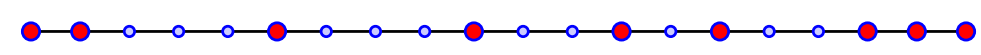

Figure 2. A configuration.

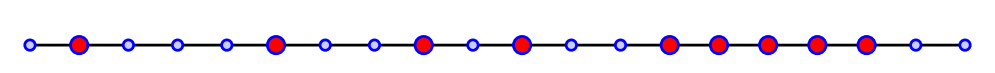

Figure 3. A forbidden configuration.

A configuration or broken necklace is one resulting from one of the $r+$ 1 cuts to the left, right or in between the medallion (as described in the motivational introduction). Figure 2 shows a configuration and Figure 3 depicts a forbidden cut.

Label $n$ vertices as $\{1,2, \cdots, n-1, n\}$. The neighbors of the vertex $i$ are $i-1$ and $i+1$ for $2 \leq i \leq n-1$; the single vertex 2 for $i=1$ and the single vertex $n-1$ for $i=n$. Configurations consist of a linear array of $n$ vertices, $k$ of which are marked or painted red. The marked vertices are either isolated, that is, its neighbors are not marked or connected, that is, the sequence of vertices $\{i, i+1, i+2, \cdots, j\}$ are all marked. In the latter case, it must be the case that $i=1$ or $j=n$; that is, connected marked vertices contain 1 or $n$.

Question 1. Determine the number $Z_{k}(n)$ of configurations up to symmetry.

The problem above, sans restriction, may be interpreted as a periodic chain made of two kinds of beads. The classical result on counting all necklaces with $n$ beads is given by MacMahon formula

$$
N(n)=\frac{1}{n} \sum_{d \mid n} \varphi(d) 2^{n / d},
$$

where the summation runs through all divisors $d$ of $n$, and $\varphi(d)$ is the Euler totient function counting the numbers $1,2, \ldots, d$ relatively prime to $d$.

Onofri et al [5] introduced a supersymmetric quantum mechanical model for a system whose degrees of freedom are bosonic and fermionic (creation and destruction) operator matrices. In the Hilbert space of this model, the vectors can be put in one-to-one correspondence with binary necklaces (or periodic linear sequences), where 1 and 0 represent fermionic and bosonic matrices respectively. Pauli's exclusion principle provides a Fermi statistics which projects out a subset among all necklaces. As such the resulting space allows a genuine depiction of supersymmetry.

The Hilbert space of the above model is generated by states due to single trace operators. Since trace is cyclically symmetric, all length $n$ necklaces related by cyclic shifts are identified as having the same state of $n$ quanta. The Pauli principle is reflected in the fact that the fermionic operators are Grassmannian variables, hence they anti-commute; while their bosonic counterparts commute freely as scalars. The number of necklaces 
is enumerated by MacMahon's formula (1.1). Some of these are excluded as a result of anti-symmetry of planar states, dictated by supersymmetry. The following serves as an illustrative example with $n=4$. There is a total of 6 necklaces listed here as linear sequences of period 4, namely

0000, 0001, 0011, 0101, 0111, 1111.

Supersymmetry breaks up this family into forbidden and allowed necklaces as follows. Given a sequence, start shifting a digit from right to left (prefix to suffix) and repeat until the original sequence is recovered. Every time a fermionic operator (that is, a 1) crosses another then a sign change must be registered because of anti-commuting; each bosonic operator (i.e., a 0) shifts around without any effect. At the end of the procedure, if the sequence becomes its own negative then call it forbidden, otherwise it is allowed. One of the main results of [5] predicts

$$
N_{\text {allowed }}(n)=\frac{1}{n} \sum_{\substack{d \mid n \\ d \text { odd }}} \varphi(d) 2^{n / d}, \text { and } N_{\text {forbidden }}(n)=\frac{1}{n} \sum_{\substack{d \mid n \\ d \text { even }}} \varphi(d) 2^{n / d} .
$$

Let us take a look at each of the above sequences now: 0000 stays the same; $0001 \rightarrow 1000 \rightarrow 0001 ; 0011 \rightarrow-1001 \rightarrow+1100 \rightarrow 0011 ; 0101 \rightarrow$ $-1010 \rightarrow-0101 ; 0111 \rightarrow+1011 \rightarrow+1101 \rightarrow+1110 \rightarrow 0111 ; 1111 \rightarrow$ -1111 . Therefore there are four allowed $\{0000,0001,0011,0111\}$ and two forbidden $\{0101,1111\}$ sets of necklaces. This agrees with

$$
N_{\text {allowed }}=\frac{1}{4}\left[\varphi(1) 2^{4}\right]=4, \quad \text { and } \quad N_{\text {forbidden }}=\frac{1}{4}\left[\varphi(2) 2^{2}+\varphi(4) 2^{1}\right]=2 .
$$

The interested reader should find the complete story in [5].

The central object of the work presented here is a sequence of numbers labeled necklace binomial coefficients $\left(\begin{array}{l}t \\ k\end{array}\right)_{\mathfrak{R}}$. These coefficients have properties similar to the usual binomial coefficients: recurrences (Corollary 2.7), symmetries (Corollary 2.12) and an explicit formula in terms of the binomial coefficients (Theorem 2.8). Section 3 discusses a surprising result on the zeros of the necklace polynomial $N_{t}(y)$ (this is the generating function of $\left(\begin{array}{l}t \\ k\end{array}\right)_{\mathfrak{N}}$ ). It turns out that all its zeros are inside an elliptic curve. Moreover, this curve is the same for all values of $t$. Section 4 presents arithmetical and geometrical properties of these polynomials via generating function methods. The necklace binomial coefficients also appear in a physical model of Onofri et al [5] and in the study of symmetries of paraffin molecules in [4].

\section{THE NUMBER OF CONFIGURATIONS}

In this section the counting problem from the Introduction is rephrased and solved. The current format as well as the original formulation will be 
used interchangeably:

determine the number $Z_{k}(n)$ of painting $k$ points in red from a linear array of $n$ of them, with the condition that consecutive red points can only appear at the beginning or at end of the array. Moreover, arrays that are reflections of each other should be counted only once.

In order to determine the number of configurations $Z_{k}(n)$ it is convenient to begin with a simpler count.

Proposition 2.1. Let $f_{k}(n)$ be the number of arrangements of $n$ vertices with $k$ marked vertices, no consecutive marked ones where reflections are not identified. Then

$$
f_{k}(n)=\left(\begin{array}{c}
n-k+1 \\
k
\end{array}\right)
$$

Proof. Each such arrangement can be obtained by placing the $k$ marked vertices and choosing $k-1$ places to separate them. The count is obtained by eliminating the separating spaces.

Reduced configurations. The next step is to count those configurations obtained by cutting the necklace exactly on one side of the medallion. These produce linear arrays where clustered vertices appear either at the beginning or at the end of the array. Invoking symmetry, only those with the medallion at the left will be considered.

Definition 2.1. The function $\beta_{k}(n)$ denotes the number of linear arrays obtained by cutting a necklace with $n$ vertices and $k$ marked vertices with a medallion at the left of the array.

Theorem 2.8 provides an expression for the function $\beta_{k}(n)$ and Theorem 2.16 provides a formula for $Z_{k}(n)$.

Definition 2.2. Let $g_{k}(n)$ be the number of arrangements of $n$ vertices with $k$ marked points, no two being consecutively marked and identifying symmetric pairs.

Example 2.2. A numerical example of $g_{k}(n)$ is given here. Take $n=4$ and $k=2$. From the pairs $\{12,13,14,23,24,34\}$ eliminate $\{12,23,34\}$ for being consecutive somewhere. This leaves $\{13,14,24\}$. The pairs are now considered modulo 5, so that 24 is identified with 13 (the same as 31 ). The final allowed list is $\{13,14\}$ showing that $g_{2}(4)=2$.

Theorem 2.3. The function $\beta_{k}(n)$ satisfies

$$
\beta_{k}(n)=g_{k}(n)+\sum_{r=2}^{k} f_{k-r}(n-r-1) .
$$


Proof. Separate the different configurations into two groups: those with no consecutive marked points and those with at least two consecutive ones that are marked. The first type is counted by $g_{k}(n)$. Observe that if a certain arrangement has two or more adjacent marked vertices, then the remaining marked ones have no restrictions due to symmetry. In other words, reflection only imposes limitations if the configuration has no adjacent marked vertices in it.

The number of possible consecutive marked points is given by the size of the medallion. If this size is $r$, with $2 \leq r \leq k$, then drop $r+1$ places from the configuration ( $r$ for the medallion and one more at the right-end of it). This leaves a total of $n-r-1$ spaces where to place $k-r$ marked vertices.

The next step is the enumeration of $g_{k}(n)$. This group is divided into three disjoint subclasses, those with (1) both ends are marked, (2) both ends are unmarked and (3) only the left end is marked. In the first class drop the vertices at positions $1,2, n-1$ and $n$ and observe that the remaining $n-4$ vertices have $k-2$ marked ones and no further restrictions. Therefore there are $g_{k-2}(n-4)$ such arrangements. Similarly, the class (2) has $g_{k}(n-2)$ elements. Finally, in class (3), drop the first two vertices and the last one that is not marked. The remaining $n-3$ vertices have no symmetry restriction. The latter are counted by $f_{k-1}(n-3)=\left(\begin{array}{c}n-k-1 \\ k-1\end{array}\right)$ such arrangements. This gives the relation

$$
g_{k}(n)=g_{k}(n-2)+g_{k-2}(n-4)+\left(\begin{array}{c}
n-k-1 \\
k-1
\end{array}\right) .
$$

Theorem 2.4. Let $n=m+2 k-1$ and define $\bar{g}_{k}(m):=g_{k}(m+2 k-1)$. Then $\bar{g}_{k}$ satisfies

$$
\bar{g}_{k}(m)=\bar{g}_{k-2}(m)+\bar{g}_{k}(m-2)+\left(\begin{array}{c}
m+k-2 \\
k-1
\end{array}\right) .
$$

Proof. Observe that any valid arrangement counted by $g_{k}(n)$ must satisfy $n \geq 2 k-1$. The rest is elementary.

The next result was obtained from experimental data generated by (2.4).

Example 2.5. The function $\bar{g}_{k}(m)$ is computed for $0 \leq m \leq 3$ :

$$
\bar{g}_{k}(0)=1, \bar{g}_{k}(1)=\left\lfloor\frac{k+2}{2}\right\rfloor, \bar{g}_{k}(2)=\left\lfloor\frac{(k+2)^{2}}{4}\right\rfloor
$$

and

$$
\bar{g}_{k}(3)=\sum_{j=0}^{k}(-1)^{k-j}\left\{\sum_{i=0}^{j}\left\lfloor\frac{j+2}{2}\right\rfloor+\left(\begin{array}{c}
j+1 \\
2
\end{array}\right)\right\} .
$$


Definition 2.6. The relation (2.4) attains a cleaner form by introducing the necklace binomial coefficients

$$
\left(\begin{array}{l}
t \\
k
\end{array}\right)_{\mathfrak{R}}:= \begin{cases}g_{k}(t+k-1) & \text { for } 0 \leq k \leq t \\
0 & \text { otherwise. }\end{cases}
$$

The next result is a restatement of Theorem 2.4 ,

Corollary 2.7. The necklace binomial coefficient satisfies the Pascal-type relation

$$
\left(\begin{array}{l}
t \\
k
\end{array}\right)_{\mathfrak{N}}=\left(\begin{array}{l}
t-2 \\
k-2
\end{array}\right)_{\mathfrak{N}}+\left(\begin{array}{l}
t-2 \\
k-1
\end{array}\right)+\left(\begin{array}{c}
t-2 \\
k
\end{array}\right)_{\mathfrak{N}}
$$

The evaluation of the necklace binomial coefficients is now easy to guess and establish using (2.8).

Theorem 2.8. For $0 \leq k \leq t$, it holds that

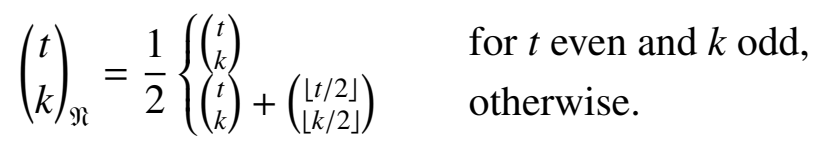

Moreover,

$$
\beta_{k}(t)=\left(\begin{array}{c}
t-k+1 \\
k
\end{array}\right)_{\mathfrak{N}}+\sum_{r=2}^{k}\left(\begin{array}{l}
t-k \\
r-2
\end{array}\right) .
$$

Table 2 shows the values of the necklace coefficients:

\begin{tabular}{|c||c|c|c|c|c|c|c|c|c|c|c||}
\hline$t / k$ & 0 & 1 & 2 & 3 & 4 & 5 & 6 & 7 & 8 & 9 & 10 \\
\hline \hline 1 & 1 & 1 & & & & & & & & & \\
2 & 1 & 1 & 1 & & & & & & & & \\
3 & 1 & 2 & 2 & 1 & & & & & & & \\
4 & 1 & 2 & 4 & 2 & 1 & & & & & & \\
5 & 1 & 3 & 6 & 6 & 3 & 1 & & & & & \\
6 & 1 & 3 & 9 & 10 & 9 & 3 & 1 & & & & \\
7 & 1 & 4 & 12 & 19 & 19 & 12 & 4 & 1 & & & \\
8 & 1 & 4 & 16 & 28 & 38 & 28 & 16 & 4 & 1 & & \\
9 & 1 & 5 & 20 & 44 & 66 & 66 & 44 & 20 & 5 & 1 & \\
10 & 1 & 5 & 25 & 60 & 110 & 126 & 110 & 60 & 25 & 5 & 1 \\
\hline
\end{tabular}

This array is known as the Losanitsch's triangle and information about it can be found in Entry A034851 of Neil Sloane's Encyclopedia of Integer Sequences.

A series of elementary consequences of (2.9) are presented next. 
Corollary 2.9. The row-sum identity

$$
\sum_{k=0}^{t}\left(\begin{array}{l}
t \\
k
\end{array}\right)_{\mathfrak{R}}=2^{t-1}+2^{\lfloor(t-1) / 2\rfloor}
$$

holds.

The next statements employ the Fibonacci numbers $F_{n}$, defined by the relation $F_{n}=F_{n-1}+F_{n-2}$ with initial conditions $F_{0}=F_{1}=1$ and the Lucas numbers $L_{n}$ defined by the same recurrence and with initial conditions $L_{0}=$ $2, L_{1}=1$.

Corollary 2.10. Let $F_{n}$ and $L_{n}$ as above. Denote $\tilde{t}:=\lfloor t / 2\rfloor+2+(-1)^{t+1}$. Then

$$
\sum_{k=0}^{t} \beta_{k}(t)=\frac{1}{2}\left(L_{t+2}+F_{\tilde{t}}\right)-1 .
$$

Corollary 2.11. The generating functions

$$
\begin{aligned}
& \sum_{k=0}^{t}\left(\begin{array}{l}
t \\
k
\end{array}\right)_{\mathfrak{N}} y^{k}=\frac{1}{2}(1+y)^{t}+\frac{1}{2}\left(1+y^{2}\right)^{\lfloor t / 2\rfloor}(1+y)^{t \bmod 2}, \\
& \sum_{t \geq 0}\left(\begin{array}{l}
t \\
k
\end{array}\right)_{\mathfrak{R}} x^{t}=\frac{(1+x)^{\lfloor(k+1) / 2\rfloor}+(1-x)^{\lfloor(k+1) / 2\rfloor}}{2(1-x)^{\lceil(k+1) / 2\rceil}\left(1-x^{2}\right)^{\lfloor(k+1) / 2\rfloor}}
\end{aligned}
$$

and

$$
\sum_{t, k \geq 0}\left(\begin{array}{l}
t \\
k
\end{array}\right)_{\mathfrak{R}} x^{t} y^{k}=\frac{1}{2(1-x-y)}+\frac{2+x}{2\left(1-x^{2}-y\right)}
$$

hold.

Corollary 2.12. The necklace binomial coefficients are symmetric, that is,

$$
\left(\begin{array}{l}
t \\
k
\end{array}\right)_{\mathfrak{N}}=\left(\begin{array}{c}
t \\
t-k
\end{array}\right)_{\mathfrak{R}}
$$

for $0 \leq k \leq t$.

Corollary 2.13. The function $\bar{g}$ is symmetric; that is,

$$
\bar{g}_{k}(m)=\bar{g}_{m}(k) \text {. }
$$

Proof. This is a restatement of (2.16). An alternative proof of the symmetry (2.17) is obtained from the recurrence (2.4). Simply express it in two different forms

$$
\begin{aligned}
\bar{g}_{k}(m)-\bar{g}_{k-2}(m) & =\bar{g}_{k}(m-2)+\left(\begin{array}{c}
m+k-2 \\
k-1
\end{array}\right), \\
\bar{g}_{k}(m)-\bar{g}_{k}(m-2) & =\bar{g}_{k-2}(m)+\left(\begin{array}{c}
m+k-2 \\
k-1
\end{array}\right) .
\end{aligned}
$$


The result now follows by induction and the symmetry of the binomial coefficients.

The next theorem provides a combinatorial proof of the symmetry rule (2.17).

Theorem 2.14. The symmetry $\bar{g}_{k}(m)=\bar{g}_{m}(k)$ holds.

Proof. The assertion amounts to $g_{k}(m+2 k-1)=g_{m}(k+2 m-1)$. Take a linear array of $n$ nodes and its 2-coloring (red $r$ or white $w$ ). By definition, $g_{k}(n)$ enumerates all possible ways of coloring $k$ nodes in red with the rule: (1) no two reds are consecutive; (2) two such arrays are equivalent if they relate by reflection. According to (1), it must be that the first $k-1$ reds are each followed by white. Thus, any selection of $k$ reds can be interpreted as choosing the $(k-1)$ pairs $r w$ and a free $r$. For each pair $r w$, trim-off the $w$ as well as its sitting node. That means, when $n=m+2 k-1$ then the number of nodes reduces to $m+k$ and hence $g_{k}(m+2 k-1)$ induces an equivalent counting of $(m+k)$-nodes of which $k$ are red (note: rule (1) is absent but rule (2) stays). Similarly, $g_{m}(k+2 m-1)$ tantamount to the counting of $(m+k)$-nodes of which $m$ are white. But, it is obvious that coloring $k$ nodes red on an $(m+k)$-array is equivalent to the coloring of $m$ nodes in white. This gives the required bijection. The proof is complete.

Example 2.15. This example demonstrates the above proof; i.e. $g_{k}(m+2 k-$ $1)=g_{m}(k+2 m-1)$. Take $m=2$ and $k=3$. Then, $g_{3}(7)$ and $g_{2}(6)$ count respectively the cardinality of sets

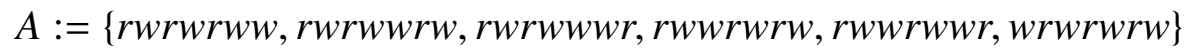

and

$$
B:\{r w r w w w, r w w r w w, w r w r w w, r w w w r w, w r w w r w, r w w w w r\} .
$$

The set $B$ after color-swapping turns to

$$
B_{1}:=\{w r w r r r, w r r w r r, r w r w r r, w r r w r, r w r r w r, w r r r r w\} .
$$

The two sets $A$ and $B_{1}$ are now mapped ( $w$-trimmed and $r$-trimmed, respectively) to

$$
A_{1}:=\{r r r w w, r r w r w, r r w w r, r w r r w, r w r w r, w r r r w\},
$$

and

$$
B_{11}:=\{w w r r r, w r w r r, r r w r r, w r r w r, r w r w r, w r r w\} .
$$

The bijection between $A_{1}$ and $B_{11}$ is clearly exposed; that is, reflect $B_{11}$ to get the set

$$
B_{111}:=\{r r r w w, r r w r w, r r w r r, r w r r w, r w r w r, w r r r w\} .
$$

The full counting solution to the configuration problem is presented next. 
Theorem 2.16. The total number $Z_{k}(t)$ of possible linear configurations of $k$ diamonds (with or without a medallion) on $t$ nodes is given by

$$
Z_{k}(t)=\sum_{j \geq 0}\left(\begin{array}{c}
t-k-1 \\
k-2 j
\end{array}\right)_{\mathfrak{R}}+\sum_{j \geq 0}\left\lfloor\frac{j+1}{2}\right\rfloor\left(\begin{array}{c}
t-k-1 \\
k-j
\end{array}\right) .
$$

Proof. Catalog the diamonds according to whether they are: (1) an equal number of clusters; (2) unequal number of clustered diamonds on the two end-nodes. However many are remaining to be mounted in the interior, case (1) is affected by the reflection but those in case (2) are not. It follows that the first case is enumerated by the function $g_{k}(t)$ (equivalently, by necklace binomials) while the function $f_{k}(t)$ is the right choice for the second category. The details are omitted.

The necklace coefficients are given as Entry A005994 in Neil Sloane's Encyclopedia of Integer Sequences. The reader will find there information on the connection between $\left(\begin{array}{l}t \\ k\end{array}\right)_{\mathfrak{N}}$ and the so-called paraffin numbers. The chemist S. M. Losanitsch studied in [4] the so-called alkane numbers (called here the necklace numbers) in his investigation of symmetries manifested by rows of paraffin (hydrocarbons). In the molecule of an alkane (also known as a paraffin), for $n$ carbon atoms there are $2 n+2$ hydrogen atoms (i.e. the form $\mathrm{C}_{n} \mathrm{H}_{2 n+2}$ ). Each carbon atom $C$ is linked to four other atoms (either $C$ of $H$ ); each hydrogen atom is joined to one carbon atom. The figures in the Appendix show all possible alkane bonds for $1 \leq n \leq 5$. There are 1, 1, 1, 2, 3 possible alignments, respectively.

A geometric interpretation. Given a finite group $G$, it is a classical problem to find the generators of the ring of polynomial invariants under the action of $G$. The Molien series $M(z ; G)$ is the generating function that counts the number of linearly independent homogeneous polynomials of a given total degree $d$ that are invariants for $G$. It is given by

$$
M(z ; G)=\frac{1}{|G|} \sum_{g \in G} \frac{1}{\operatorname{det}(I-z g)}=\sum_{i=0}^{\infty} b_{i} z^{i}
$$

Thus, the coefficients $b_{i}$ record the number of linearly independent polynomials of total degree $i$.

Now assume $k=2 m-1$. Then (2.14) becomes

$$
\sum_{i \geq 0}\left(\begin{array}{c}
i+2 m-1 \\
2 m-1
\end{array}\right)_{\mathfrak{R}} z^{i}=\frac{1}{2} \frac{1}{(1-z)^{2 m}}+\frac{1}{2} \frac{1}{\left(1-z^{2}\right)^{m}} .
$$


This is recognized as

$$
\frac{1}{2} \frac{1}{(1-z)^{2 m}}+\frac{1}{2} \frac{1}{\left(1-z^{2}\right)^{m}}=\frac{1}{|G|} \sum_{g \in G} \frac{1}{\operatorname{det}\left(\mathbf{1}_{2 m}-z g\right)},
$$

where $G$ is the symmetric group $S_{2}$ and the summation runs through the $2 m$ dimensional group representation of the elements $g$ in $G L_{2 m}(\mathbb{C})$. The argument below shows that the series is indeed a Molien series for the ring of invariants under the action of $S_{2}$. More specifically, the ring of invariants under consideration is $\mathbb{C}[X ; Y]^{\sim 2}$ where $X=\left(x_{1}, \ldots, x_{m}\right)$ and $Y=\left(y_{1}, \ldots, y_{m}\right)$. The action is given by $x_{l} \mapsto y_{l}$ for $l=1, \ldots, n$.

Let $\sigma$ be the matrix $\sigma=\left(\begin{array}{ll}0 & 1 \\ 1 & 0\end{array}\right)$ and let $\pi$ be the tensor product $\pi=\sigma \otimes \mathbf{1}_{m}$ resulting in a $2 m \times 2 m$ matrix which has four blocks of size $m \times m$ with the off-diagonal blocks being the identity matrix and the diagonals blocks being zero. The matrix group generated by $\pi$ in $G L_{2 m}$ is $S_{2}$. Consequently,

$$
\operatorname{det}\left(\mathbf{1}_{2 m}-z \pi^{2}\right)=\operatorname{det}\left(\mathbf{1}_{2 m}-z \mathbf{1}_{2 m}\right)=(1-z)^{2 m}
$$

and $\operatorname{det}\left(\mathbf{1}_{2 m}-z \pi\right)=\operatorname{det}\left(\rho \otimes \mathbf{1}_{2 m}\right)$, with $\rho=\left(\begin{array}{cc}1 & -z \\ -z & 1\end{array}\right)$. Since $\operatorname{det}(A \otimes B)=$ $\operatorname{det}(A)^{m} \operatorname{det}(B)^{m}$, it must be that $\operatorname{det}\left(\mathbf{1}_{2 m}-z \pi\right)=\left(1-z^{2}\right)^{m}$.

These observations are summarized in the next statement.

Theorem 2.17. Consider the action of $\mathbb{Z}_{2}$ on $\mathbb{C}\left[x_{1}, \cdots, x_{m}, y_{1}, \cdots, y_{m}\right]$ given by $x_{l} \mapsto y_{l}$. Then, the number of linearly independent invariant polynomials of total degree $i$ is given by the necklace binomial coefficient $\left(\begin{array}{c}i+2 m-1 \\ 2 m-1\end{array}\right)_{\mathfrak{N}}$.

\section{THE NECKLACE POLYNOMIALS}

In this section we discuss properties of the necklace polynomials defined by

$$
N_{t}(y)=\sum_{k=0}^{t}\left(\begin{array}{l}
t \\
k
\end{array}\right)_{\mathfrak{R}} y^{k}
$$

The explicit formula

$$
N_{t}(y)=\frac{1}{2}(1+y)^{t}+\frac{1}{2}\left(1+y^{2}\right)^{\lfloor t / 2\rfloor}(1+y)^{t \bmod 2}
$$

is given in 2.13). 
Example 3.1. The first few values of $N_{t}(y)$ are given by

$$
\begin{aligned}
& N_{1}(y)=1+y \\
& N_{2}(y)=1+y+y^{2} \\
& N_{3}(y)=N_{1}(y) N_{2}(y) \\
& N_{4}(y)=1+2 y+4 y^{2}+2 y^{3}+y^{4} \\
& N_{5}(y)=N_{1}(y) N_{4}(y) \\
& N_{6}(y)=N_{2}(y)\left(1+2 y+6 y^{2}+2 y^{3}+y^{4}\right) \\
& N_{7}(y)=N_{1}(y) N_{2}(y)\left(1+2 y+6 y^{2}+2 y^{3}+y^{4}\right) \\
& N_{8}(y)=1+4 y+16 y^{2}+28 y^{3}+38 y^{4}+28 y^{5}+16 y^{6}+4 y^{7}+y^{8} .
\end{aligned}
$$

The sequence of necklace polynomials have some interesting divisibility properties. The results presented below began with the empirical observation that, for $t$ odd, $N_{t}(y)=N_{1}(t) N_{t-1}(y)$.

Corollary 3.2. Let $j \in \mathbb{N}$ and $t \in \mathbb{N}$. Then $N_{j}(y)$ divides $N_{(2 t-1) j}(y)$.

Proof. This is a direct consequence of the explicit formula given in Theorem 3.2 .

Problem 3.3. Prove that $N_{2^{j}}(y)$ is irreducible.

Many polynomials appearing in combinatorics are unimodal; that is, there is an index $n^{*}$ such that the coefficients increase up to $n^{*}$ and decrease from that point on. A stronger property is that of logconcavity: the polynomial $P(x)=\sum_{k=0}^{n} a_{k} x^{k}$ is logconcave if $a_{k}^{2}-a_{k-1} a_{k+1} \geq 0$ for $1 \leq k \leq n-1$. The reader is referred to [2, 7] for surveys on these issues.

The explicit expression (2.9) gives an elementary proof of the next statement.

Theorem 3.4. The necklace binomial coefficients are unimodal.

Proof. The inequality

$$
\left(\begin{array}{l}
t \\
k
\end{array}\right)_{\mathfrak{N}} \leq\left(\begin{array}{c}
t \\
k+1
\end{array}\right)_{\mathfrak{N}}
$$

for $0 \leq k \leq\lfloor t / 2\rfloor$ and the symmetry of the necklace binomial coefficients, established in Theorem 2.12, give the result.

Theorem 3.5. The polynomial $N_{t}(y)$ is logconcave.

Proof. Use (2.9) and separate cases according to the parity of $t$ and $k$.

Problem 3.6. Let $\mathfrak{L}\left\{a_{n}\right\}:=\left\{a_{n}^{2}-a_{n-1} a_{n+1}\right\}$ be an operator defined on nonnegative sequences. Therefore, a polynomial $P(x)$ is logconcave if $\mathfrak{L}$ maps 
its coefficients into a nonnegative sequence. The polynomial $P$ is called $k$ logconcave if $\mathfrak{Q}^{(j)}(P)$ is nonnegative for $0 \leq j \leq k$. A sequence is called infinitely logconcave if it is $k$-logconcave for every $k \in \mathbb{N}$.

A recent result of $\mathrm{P}$. Brändén [1] proves that if a polynomial $P$ has only real and negative zeros, then the sequence of its coefficients is infinitely logconcave. The sequence of binomial coefficients satisfies this property.

The question proposed here is to prove that $N_{t}(y)$ is infinitely logconcave.

There is a well-established connection between unimodality questions and the location of the zeros of a polynomial. For example, a polynomial with all its zeros real and negative is logconcave [8]. This motivated the computation of the zeros of $N_{t}(y)$. Figure 4 shows the zeros of $N_{100}(y)$.

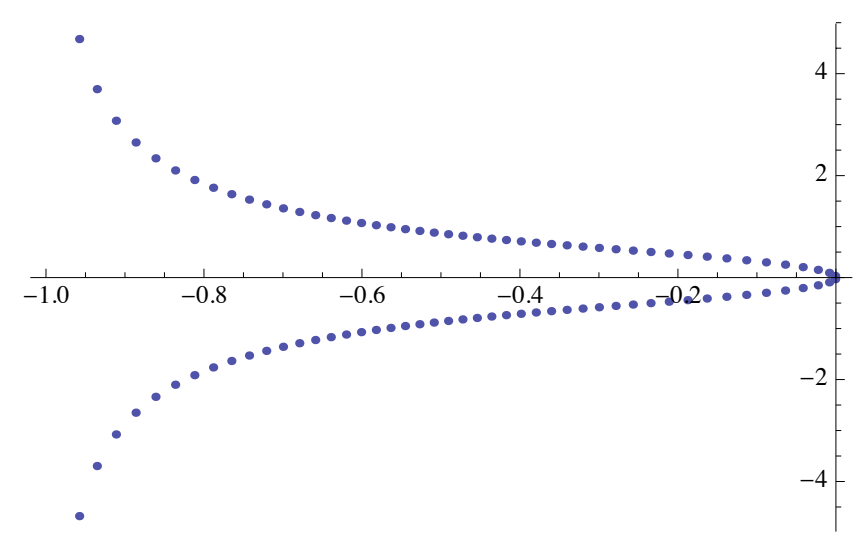

FIGURE 4. The zeros of the necklace polynomial $N_{100}(y)$.

Theorem 3.7. Let $y=a+i b$ be a root of the necklace polynomial $N_{t}(y)=0$. For $a \neq-1$, define the new coordinates $u=1 /(1+a)$ and $v=b /(1+a)$. Then $(u, v)$ is on the elliptic curve $v^{2}=u^{3}-2 u^{2}+2 u-1$.

Proof. Any zero of $N_{t}(y)$ satisfies

$$
(1+y)^{t}=- \begin{cases}\left(1+y^{2}\right)^{t / 2} & \text { if } t \text { is even } \\ \left(1+y^{2}\right)^{(t-1) / 2}(1+y) & \text { if } t \text { is odd }\end{cases}
$$

Taking the complex modulus produces $|1+y|^{4}=\left|1+y^{2}\right|^{2}$. In terms of $y=a+i b$ this equation becomes

$$
b^{2}=-\frac{a\left(a^{2}+a+1\right)}{1+a} .
$$


The transformation $1+a=1 / u$ and $b=v / u$ leads to equation

$$
v^{2}=u^{3}-2 u^{2}+2 u-1=(u-1)\left(u^{2}-u+1\right),
$$

as claimed.

Note 3.8. The collection of points on an elliptic curve $\mathfrak{E}$, such as $(3.6)$, has been the subject of research since the 18th century. The general equation of such a curve is written as

$$
y^{2}+a_{1} y=x^{3}+a_{2} x^{2}+a_{4} x+a_{6}
$$

and if $x, y \in P\left(\mathbb{C}^{2}\right)$, the complex projective space, then $\mathbb{E}$ is a torus. The addition of this torus is expressed on the cubic in a geometric form: to add $P_{1}$ and $P_{2}$, form the line joining them and define $P_{3}:=P_{1} \oplus P_{2}$ as the reflection of the third point of intersection of this line with the cubic curve. This addition rule is expressed in coordinate form: the general formula given in [6]. Let $P_{1}=\left(x_{1}, y_{1}\right)$ and $P_{2}=\left(x_{2}, y_{2}\right)$. Define

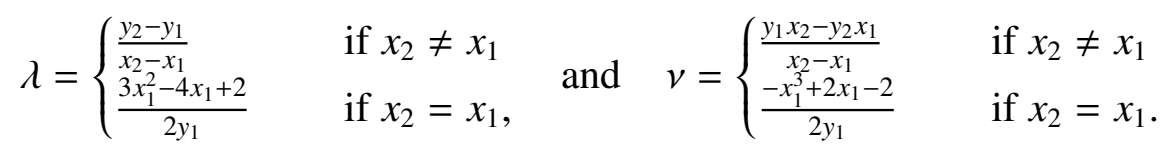

The $P_{3}=\left(x_{3}, y_{3}\right)$ is given by

$$
x_{3}=\lambda^{2}+2-x_{1}-x_{2} \text { and } y_{3}=-\lambda x_{3}-v .
$$

Aside from the point $P_{0}=(1,0)$, the table below shows a collection of points on the curve $\mathfrak{E}$ obtained using Mathematica. The notation

$$
\begin{array}{r}
\gamma=\sqrt{3+2 \sqrt{3}}, \delta=\sqrt{\sqrt{5}-2}, \tau=\sqrt{24+14 \sqrt{3}}, \sigma=2 \sqrt{2(11+5 \sqrt{5})}, \\
\omega_{1}=2+\sqrt{3}, \omega_{2}=2(3+\sqrt{5}), \omega_{3}=3+2 \sqrt{3}
\end{array}
$$

is employed.

The notation necklace point refers to a point $(u, v)$ on the elliptic curve $\mathbb{E}$ that is produced by the zero $y=a+i b$ of a necklace polynomial via the transformation $1+a=1 / u$ and $b=v / u$. The addition of two necklace points sometimes yields another one. For instance, $P_{1} \oplus P_{1}=P_{0}$ and $2 P_{3}:=$ $P_{3} \oplus P_{3}=P_{2}$. On the other hand, the set of necklace points is not closed under addition:

$P_{1} \oplus P_{7}=\frac{1}{2}(7+3 \sqrt{5}+\sqrt{66+30 \sqrt{5}})-\frac{I}{2}(21+9 \sqrt{5}+\sqrt{30(29+13 \sqrt{5})})$.

The minimal polynomial for this number is $y^{8}-28 y^{7}+1948 y^{6}-5236 y^{5}+$ $4858 y^{4}-3988 y^{3}+7156 y^{2}-6040 y+2245$. This polynomial does not divide a $N_{t}(y)$ for $1 \leq t \leq 1000$. It is conjectured that it never does. 


\begin{tabular}{||c|c|c|c||}
\hline Name & $u$ & $v$ & Root of $N_{t}(y)=0$ \\
\hline$P_{1}$ & 2 & $-\sqrt{3}$ & 2 \\
$P_{2}$ & 2 & $+\sqrt{3}$ & 2 \\
$P_{3}$ & $\omega_{1}-\gamma$ & $\omega_{3}-\tau$ & 6 \\
$P_{4}$ & $\omega_{1}-\gamma$ & $-\omega_{3}+\tau$ & 6 \\
$P_{5}$ & $\omega_{1}+\gamma$ & $-\omega_{3}-\tau$ & 6 \\
$P_{6}$ & $\omega_{1}+\gamma$ & $\omega_{3}+\tau$ & 6 \\
$P_{7}$ & $(1+\delta) \omega_{2}$ & $\omega_{2}+\sigma$ & 4 \\
$P_{8}$ & $(1+\delta) \omega_{2}$ & $-\left(\omega_{2}+\sigma\right)$ & 4 \\
$P_{9}$ & $(1-\delta) \omega_{2}$ & $\omega_{2}-\sigma$ & 4 \\
$P_{10}$ & $(1-\delta) \omega_{2}$ & $-\left(\omega_{2}-\sigma\right)$ & 4 \\
\hline
\end{tabular}

TABLE 1. Some points on the elliptic curve $\mathfrak{E}$.

Note 3.9. Equation (3.5) shows that any root of $N_{t}(y)$ must satisfy $-1 \leq$ $\operatorname{Re} y \leq 0$. Observe that $y=0$ is never a root.

Note 3.10. The change of variables $u \mapsto u+1$ transforms the curve $\mathfrak{E}$ into the form $v^{2}=u^{3}+u^{2}+u$. This curve appears as $48 a 4$ in Cremona's table of elliptic curves, available at

http://www .ma.utexas.edu/users/tornaria/cnt/cremona.html? conductor $=48$

The discriminant of the cubic is negative. Therefore the curve has a single real component. This is seen in Figure 4.

Problem 3.11. The zeros of the polynomial $N_{t}(y)$ are algebraic numbers lying on the elliptic curve $(3.5)$. The points on that curve with algebraic coordinates form a subgroup $\mathcal{A}$ under the addition described above. The question is to characterize in $\mathcal{A}$ the set coming from necklace points.

\section{Necklaces AND THEIR PROGENY}

This section explores the enumeration of certain special necklaces and their generating functions. The latter is applied to the computation of some Molien series. A circuit graph is a graph consisting of $n$ vertices placed on a circle with some of them colored by red.

Proposition 4.1. The total number of $n$-bead (circular) necklaces on which a red-red string is forbidden is given by

$$
W(n)=\frac{1}{n} \sum_{d \mid n} \varphi\left(\frac{n}{d}\right) L_{d} .
$$

Proof. A standard application of Burnside's lemma. 
Example 4.2. For $n=p$ prime, formula (4.1) gives

$$
W(p)=\frac{(p-1)+L_{p}}{p} .
$$

It follows that $L_{p} \equiv 1 \bmod p$. Similarly, for $n=p^{2}, 4.1$ gives

$$
p^{2} W\left(p^{2}\right)=L_{p^{2}}+(p-1) L_{p}+p(p-1) .
$$

It follows that

$$
L_{p^{2}} \equiv L_{p}+1 \bmod p^{2} .
$$

These are well-known results [3].

A more distinguishing count is provided by defining $W_{k}(n)$ to be the number of $n$-bead necklaces on which a red-red string is forbidden, consisting of exactly $k$ red beads. In order to accomodate the possibility that $k=0$, we define $W_{0}(n):=1$ (this is justifiable since $W_{0}(n)=\frac{1}{n} \sum_{d \mid n} \varphi(d)=1$ ).

Theorem 4.3. The function $W_{k}(n)$ is given by

$$
W_{k}(n)=\frac{1}{n-k} \sum_{d \mid n, k} \varphi(d)\left(\begin{array}{c}
\frac{n}{d}-\frac{k}{d} \\
\frac{k}{d}
\end{array}\right) .
$$

Proof. It follows directly from Burnside's lemma.

Corollary 4.4. The identity

$$
\sum_{k=0}^{\lfloor n / 2\rfloor} \frac{1}{n-k} \sum_{d \mid n, k} \varphi(d)\left(\begin{array}{c}
\frac{n}{d}-\frac{k}{d} \\
\frac{k}{d}
\end{array}\right)=\frac{1}{n} \sum_{d \mid n} \varphi(d) L_{n / d}
$$

holds.

Proof. The assertion follows from the combinatorial identity

$$
\sum_{k \geq 0} W_{k}(n)=W(n)
$$

Theorem 4.5. For $n \in \mathbb{N}$ define

$$
V_{d}(x)=\left(\frac{1-\sqrt{1+4 x}}{2}\right)^{d}+\left(\frac{1+\sqrt{1+4 x}}{2}\right)^{d} .
$$

Then the row-sum generating function of $W_{k}(n)$ is given by

$$
F_{n}(x):=\sum_{k=0}^{\lfloor n / 2\rfloor} W_{k}(n) x^{k}=\frac{1}{n} \sum_{d \mid n} \varphi\left(\frac{n}{d}\right) V_{d}\left(x^{n / d}\right) .
$$


Proof. The proof is based on the identity

$$
\frac{1}{m} V_{m}(x)=\sum_{k=0}^{\lfloor m / 2\rfloor} \frac{1}{m-k}\left(\begin{array}{c}
m-k \\
k
\end{array}\right) x^{k},
$$

which is easy to verify. This is applied to

$$
\begin{aligned}
\sum_{k=0}^{\lfloor n / 2\rfloor} W_{k}(n) x^{k} & =\sum_{d \mid n} \varphi(d) \sum_{k \geq 0} \frac{1}{n-d k}\left(\begin{array}{c}
\frac{n}{d}-k \\
k
\end{array}\right) x^{d k} \\
& =\sum_{d \mid n} \frac{\varphi(d)}{d} \sum_{k \geq 0} \frac{1}{\frac{n}{d}-k}\left(\begin{array}{c}
\frac{n}{d}-k \\
k
\end{array}\right) x^{d k}
\end{aligned}
$$

The result follows from here.

Example 4.6. For $p$ prime, the polynomial $F_{p}(x)$, defined in (4.9), is given by

$$
\begin{aligned}
F_{p}(x) & =\sum_{k=0}^{\lfloor p / 2\rfloor} \frac{1}{p-k}\left(\begin{array}{c}
p-k \\
k
\end{array}\right) x^{k} \\
& =\frac{(p-1) 2^{p}(1-\sqrt{1+4 x})^{p}+(1+\sqrt{1+4 x})^{p}}{p \cdot 2^{p}} .
\end{aligned}
$$

Example 4.7. Put $n=3 k+1$ in 4.3 to obtain $W_{k}(3 k+1)=\frac{1}{2 k+1}\left(\begin{array}{c}2 k+1 \\ k\end{array}\right)$, the Catalan numbers.

Example 4.8. For $n \in \mathbb{N}$, and with $L_{n}$ denoting the Lucas number,

$$
\sum_{k=0}^{\lfloor n / 2\rfloor} \frac{1}{n-k}\left(\begin{array}{c}
n-k \\
k
\end{array}\right)=\frac{1}{n} L_{n} .
$$

This is obtained from setting $x=1$ in 4.9 .

Theorem 4.9. The ordinary generating function for the diagonals of $W_{k}(n)$ is given by

$$
\sum_{n \geq k} W_{k}(n) x^{n}=\frac{1}{k} \sum_{d \mid k} \frac{\varphi(d) x^{2 k}}{\left(1-x^{d}\right)^{k / d}} .
$$

In its lowest terms, the denominator of this rational function takes the form

$$
\prod_{d \mid k}\left(1-x^{d}\right)^{\varphi(k / d)}=\prod_{d \mid k} \Phi_{d}(x)^{k / d},
$$

where $\Phi_{d}(x)$ is the $d$-th cyclotomic polynomial given in terms of the Mobius $\mu$-function as $\Phi_{d}(x)=\prod_{c \mid d}\left(1-x^{d / c}\right)^{\mu(x)}$. 
Proof. The result follows from the Taylor series expansion

$$
\frac{x^{2 m}}{m(1-x)^{m}}=\sum_{j \geq m} \frac{1}{j-m}\left(\begin{array}{c}
j-m \\
m
\end{array}\right) x^{j} .
$$

A geometric interpretation. The above generating function $\sum_{n \geq k} W_{k}(n) x^{n}$ is the Molien series $W\left(x ; \mathbb{Z}_{k}\right)$ for the ring of invariants $\mathbb{C}[X]^{\mathbb{Z}_{k}}$ where $X=$ $\left(x_{1}, \ldots, x_{k}\right)$. In this case, the group $\mathbb{Z}_{k}$ is identified with its $k$-dimensional group representation in $G L_{k}(\mathbb{C})$. More concretely, $\mathbb{Z}_{k} \cong\left\langle\mathbf{e}_{k}\right\rangle$ where $\mathbf{e}_{k}$ is the $k \times k$ permutation matrix such that $\mathbf{e}[i, j]=1$ if $j=i+1 ; \mathbf{e}[k, 1]=1$ and $\mathbf{e}[i, j]=0$, otherwise. Let $R P(d)$ be the set of positive integers less than $d$ and relatively prime to $d$. Partition the integer interval $[k]$ into the disjoint union

$$
[k]=\{1,2, \ldots, k\}=\bigcup_{d \mid k} \frac{k}{d} R P(d) .
$$

This relation is reminiscent of the well-known identity $k=\sum_{d \mid k} \varphi(d)$. Then,

$$
\begin{aligned}
W\left(x ; \mathbb{Z}_{k}\right) & =\frac{1}{\left|\mathbb{Z}_{k}\right|} \sum_{j=1}^{k} \frac{1}{\operatorname{det}\left(\mathbf{1}_{k}-x \mathbf{e}_{k}^{j}\right)} \\
& =\frac{1}{k} \sum_{d \mid k} \frac{\varphi(d)}{\operatorname{det}\left(\mathbf{1}_{k}-x \mathbf{e}_{k}^{k / d}\right)} \\
& =\frac{1}{k} \sum_{d \mid k} \frac{\varphi(d)}{\operatorname{det}\left(\left(\mathbf{1}_{d}-x \mathbf{e}_{d}\right) \otimes \mathbf{1}_{k / d}\right)} \\
& =\frac{1}{k} \sum_{d \mid k} \frac{\varphi(d)}{\operatorname{det}\left(\left(\mathbf{1}_{d}-x \mathbf{e}_{d}\right)^{k / d}\right.} \\
& =\frac{1}{k} \sum_{d \mid k} \frac{\varphi(d)}{\left(1-x^{d}\right)^{k / d}} .
\end{aligned}
$$

These findings are stated in the next result.

Proposition 4.10. The number of linearly independent homogeneous polynomials, of total degree $n$, for the ring of invariants $\mathbb{C}[X]^{\mathbb{Z}_{k}}$ equals

$$
\frac{1}{n+k} \sum_{d \mid n, k} \varphi(d)\left(\begin{array}{c}
\frac{n}{d}+\frac{k}{d} \\
\frac{k}{d}
\end{array}\right) \text {. }
$$




\section{A SAMPLE OF THE COMPUTATION OF ZEROS}

Motivated by the interesting properties of the zeros of necklace polynomials, this section presents some computational graphics showing the zeros of the polynomials $F_{n}(x)$. Figure 5 shows the location of the roots of $F_{1000}(x)$.

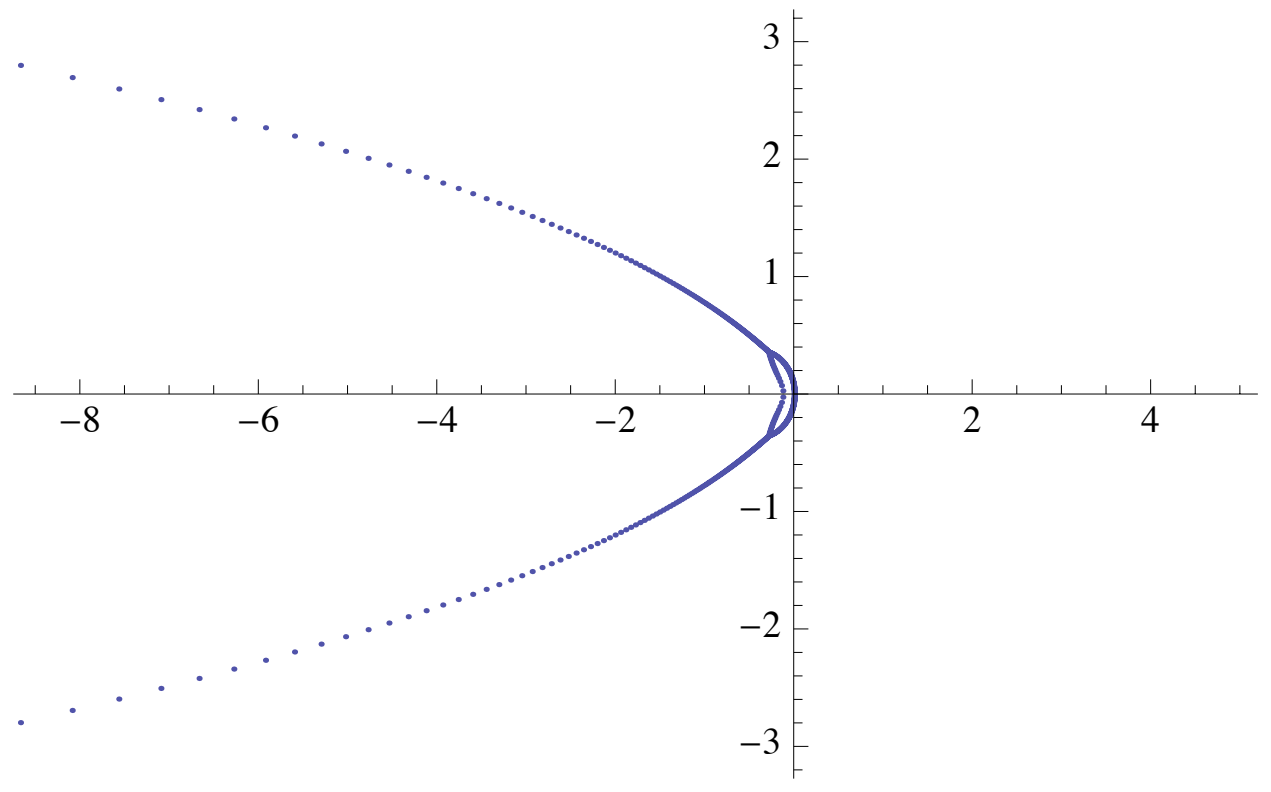

Figure 5. The zeros of $F_{1000}(x)$. 
The next four figures show a selection of regions from the set of the roots of all the polynomials $F_{n}(x)$ for $3 \leq n \leq 1000$. The caption indicates the range depicted.

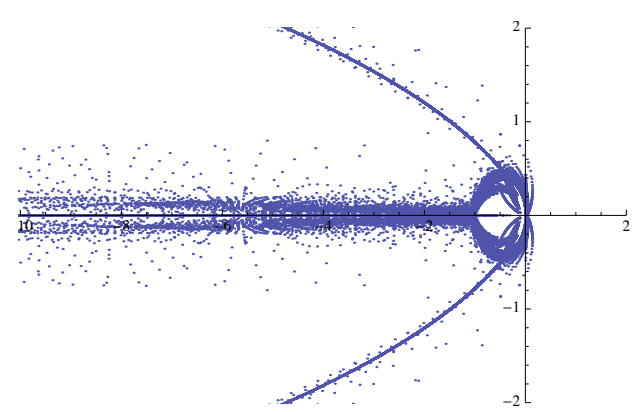

FiguRE 6. $[-10,2] \times[-2,2]$.

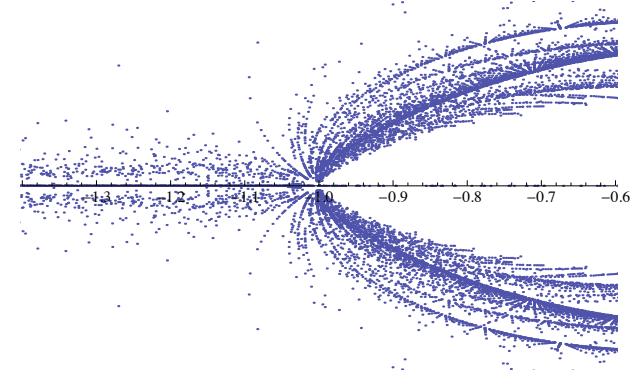

FiguRE 8. $[-1.4,-0.6] \times[-0.5,0.5]$.

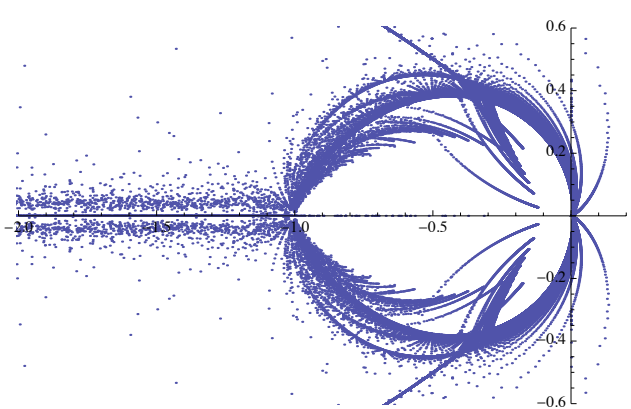

FiguRE 7. $[-2,0.2] \times[-0.6,0.6]$.

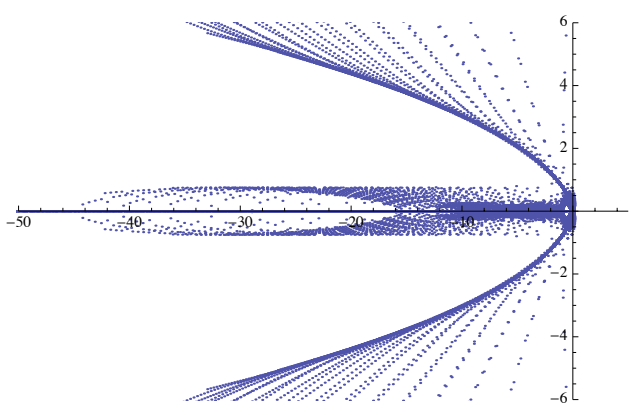

FigURE 9. $[-50,5] \times[-6,6]$.

The interesting structure depicted in figures 6 to 9 will be explored in future work.

Acknowledgments. The authors wish to thank J. Silverman for providing information on the elliptic curve mentioned in the title and to A. Ayyer and A. Waldron regarding information on the quantum mechanical systems. The authors also wish to thanks the referees for comments on an earlier manuscript. The third author was partially funded by NSF-DMS 0070567. 


\section{Appendix A. Rows of PARAFFin}

The figures show all possible alkane bonds (paraffin) $C_{n} H_{2 n+2}$ for $n=$ $1,2,3,4,5$.

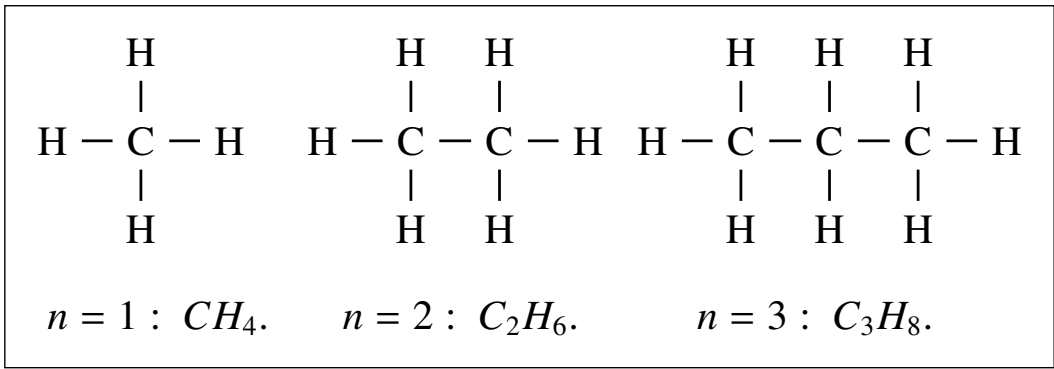

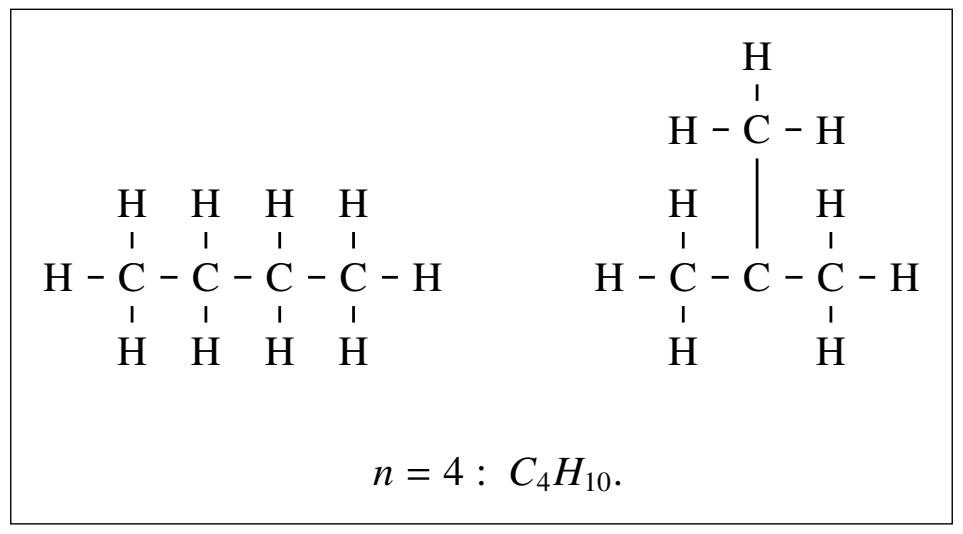

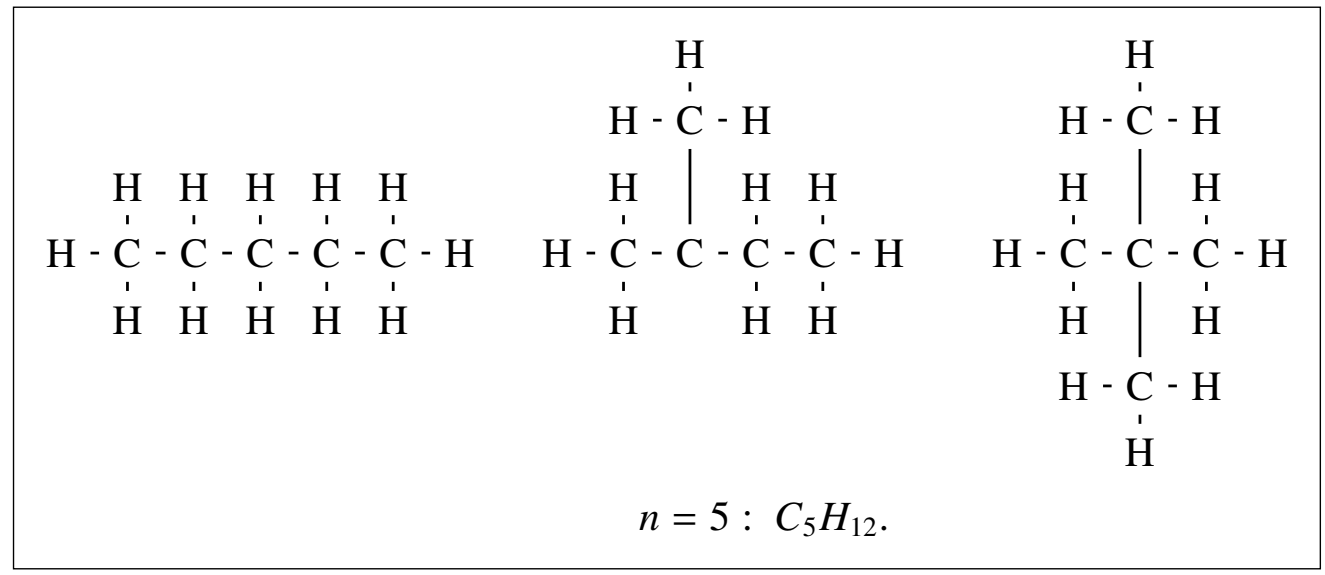




\section{REFERENCES}

[1] P. Brändén. Iterated sequences and the geometry of zeros. ArXiV: Math. C0/0909.1927, 2010.

[2] F. Brenti. Log-concave and unimodal sequences in Algebra, Combinatorics and Geometry: an update. Contemporary Mathematics, 178:71-89, 1994.

[3] G. H. Hardy, E. M. Wright; revised by D. R. Heath-Brown, and J. Silverman. An Introduction to the Theory of Numbers. Oxford University Press, 6th edition, 2008.

[4] S. M. Losanitsch. Die Isomerie-Arten bei den Homologen der Paraffin-Reihe. Chem. Ber., 30:1917-1926, 1897.

[5] E. Onofri, G. Veneziano, and J. Wosiek. Supersymmetry and Combinatorics. ArXiV: math-ph/0603082, 2010.

[6] J. H. Silverman. The Arithmetic of Elliptic Curves. Springer Verlag, New York, first edition, 1986.

[7] R. Stanley. Log-concave and unimodal sequences in Algebra, Combinatorics and Geometry. graph theory and its applications: East and West ( Jinan, 1986). Ann. New York Acad. Sci., 576:500-535, 1989.

[8] H. S. Wilf. generatingfunctionology. Academic Press, 1st edition, 1990.

Department of Mathematics, Tulane University, New Orleans, LA 70118

E-mail address: tamdeber@tulane.edu

Department of Mathematics, Tulane University, New Orleans, LA 70118

E-mail address: mcan@tulane.edu

Department of Mathematics, Tulane University, New Orleans, LA 70118

E-mail address: vhm@math. tulane. edu 